\title{
Erratum to: Sobolev estimates for the complex Green operator on CR submanifolds of hypersurface type
}

\author{
Emil J. Straube • Yunus E. Zeytuncu
}

Published online: 17 December 2014

(C) Springer-Verlag Berlin Heidelberg 2014

\section{Erratum to: Invent. Math. DOI 10.1007/s00222-014-0564-6}

We correct an inaccuracy in the references. The references from 6 to 9 were missing one of the authors, corrected references are as below.

\section{References}

[6] Boas, H.P., Straube, E.J.: Sobolev estimates for the $\bar{\partial}$-Neumann operator on domains in $\mathbb{C}^{n}$ admitting a defining function that is plurisubharmonic on the boundary. Math. Z. 206, 81-88 (1991)

[7] Boas, H.P., Straube, E.J.: Sobolev estimates for the complex Green operator on a class of weakly pseudoconvex boundaries. Commun. Partial Diff. Equ. 16(10), 1573-1582 (1991)

[8] Boas, H.P., Straube, E.J.: The Bergman projection on Hartogs domains in $\mathbb{C}^{2}$. Trans. Am. Math. Soc. 331, 529-540 (1992)

The online version of the original article can be found under doi:10.1007/s00222-014-0564-6.

\section{E. J. Straube}

Department of Mathematics, Texas A\&M University,

College Station, TX 77843, USA

e-mail: straube@math.tamu.edu

Y. E. Zeytuncu $(\bowtie)$

Department of Mathematics and Statistics, University of Michigan-Dearborn,

Dearborn, MI 48128, USA

e-mail: zeytuncu@umich.edu 
[9] Boas, H. P., Straube, E. J.: De Rham cohomology of manifolds containing the points of infinite type, and Sobolev estimates for the $\bar{\partial}$-Neumann problem. J. Geom. Anal. 3(3), 225-235 (1993) 